\title{
The double Fibonacci sequences in groups and rings
}

\author{
Ömür Deveci \\ Department of Mathematics, Faculty of Science and Letters \\ Kafkas University 36100, Turkey \\ e-mail: odeveci36@hotmail.com
}

Received: 4 July 2017

Accepted: 7 May 2018

\begin{abstract}
In this paper, we define the double Fibonacci sequence and the basic double Fibonacci sequence in groups and rings. Then we examine these sequences in finite 2-generator groups and rings. Also, we obtain the periods of the double Fibonacci sequences and the basic double Fibonacci sequences in the dihedral group $D_{2 m}$ and the ring $E$ for the generating pairs $(a, b)$ and $(b, a)$ as applications of the results obtained.
\end{abstract}

Keywords: Double Fibonacci sequence, Group, Ring, Period.

2010 Mathematics Subject Classification: 11B39, 16P10, 20 D60.

\section{Introduction}

It is well-known that the Fibonacci, the $(a, b)$-Fibonacci, the Pell and the half-companion Pell sequences are defined as follows, respectively:

$$
\begin{gathered}
F_{n}=F_{n-1}+F_{n-2} \text { for } n \geq 2 \text { in which } F_{0}=0 \text { and } F_{1}=1, \\
F_{n}^{(a, b)}=a F_{n-1}^{(a, b)}+b F_{n-2}^{(a, b)} \text { for non-zero integers } a, b \text { and } n \geq 2 \text { in which } F_{0}^{(a, b)}=0 \text { and } F_{1}^{(a, b)}=1, \\
P_{n}=2 P_{n-1}+P_{n-2} \text { for } n \geq 2 \text { in which } P_{0}=0 \text { and } P_{1}=1
\end{gathered}
$$

and

$$
H_{n+1}=2 H_{n}+H_{n-1} \text { for } n \geq 2 \text { in which } H_{0}=1 \text { and } H_{1}=1 .
$$

Let $G$ be a finite $n$-generator group and suppose that

$$
X=\{\left(x_{1}, x_{2}, \cdots, x_{n}\right) \in \underbrace{G \times G \times \cdots \times G}_{n} \mid\left\langle\left\{x_{1}, x_{2}, \cdots, x_{n}\right\}\right\rangle=G\} .
$$

We call $\left(x_{1}, x_{2}, \cdots, x_{n}\right)$ a generating $n$-tuple for $G$. 
Definition 1.1. The Fibonacci orbit of the n-generated group $G$ with respect to the generating $n$-tuple $\left(x_{1}, x_{2}, \cdots, x_{n}\right)$, written $F_{\left(x_{1}, x_{2}, \cdots, x_{n}\right)}(G)$, is the sequence $a_{i}=x_{i}, 1 \leq i \leq n, a_{n+i}=$ $\prod_{k=1}^{n} a_{i+k-1}, i \geq 1$.

Recenlty, The Fibonacci orbits of special groups have been investigated by some authors; see for example, $[1,3,4,10,11]$.

Definition 1.2. Let $R$ be a ring with identity $I$, The sequence $\left\{M_{n}\right\}$ of elements of $R$ is defined by

$$
M_{n+2}=A_{1} M_{n+1}+A_{0} M_{n}, n \geq 0
$$

in which $M_{0}, M_{1}, A_{0}$ and $A_{1}$ are arbitrary elements of $R$.

Special cases of (1) were studied in $[2,5,9,13]$.

Definition 1.3. (Tasyurdu and Deveci [12]). Let $R$ be a 2-generator ring and let $(a, b)$ be a generating pair of the ring $R$. We define the Fibonacci Polynomial-type orbit $F_{(a, b)}^{R}(x)=\left\{x_{i}\right\}$ of $(a, b)$ by

$$
x_{n+1}=b x_{n}+x_{n-1}
$$

in which $x_{0}=a$ and $x_{1}=b$.

A sequence is periodic if, after a certain point, it consists only of repetitions of a fixed subsequence. The number of elements in the shortest repeating subsequence is called the period of the sequence. In particular, if the first $n$ elements in the sequence form a repeating subsequence, then this sequence is simply periodic and its period is $n$.

Given an integer matrix $A=\left[a_{i j}\right], A(\bmod m)$ means that all entries of $A$ are modulo $m$, that is, $A(\bmod m)=\left(a_{i j}(\bmod m)\right)$. Let us consider the set $\langle A\rangle_{m}=\left\{A^{i}(\bmod m) \mid i \geq 0\right\}$. If $\operatorname{gcd}(\operatorname{det} A, m)=1$, then $\langle A\rangle_{m}$ is a cyclic group. The order of the cyclic group $\langle A\rangle_{m}$ is denoted by $\left|\langle A\rangle_{m}\right|$.

A group $D_{2 m}$ is dihedral if

$$
D_{2 m}=\left\langle a, b \mid a^{m}=b^{2}=(a b)^{2}=e\right\rangle .
$$

Note that the order of dihedral group $D_{2 m}$ is $2 m$.

For any prime $p$, up to isomorphism, the 2-generator ring $E$ of order $p^{2}$, which is not field is given by the following presentation

$$
E=\left\langle a, b \mid p a=p b=0, a^{2}=a, b^{2}=b, a b=a, b a=b\right\rangle .
$$

For more information on the ring $E$, see [7].

In this paper, we define the double Fibonacci sequence and the basic double Fibonacci sequence in groups and rings and then we give the periods of the double Fibonacci sequences in the dihedral group $D_{2 m}$ and the ring $E$ for the generating pairs $(a, b)$ and $(b, a)$. 


\section{The main results}

Define the double Fibonacci sequence $\{F(n, k)\}$ as shown:

$$
F(n, k)=F_{n+2} F_{k+1}+F_{n+1} F_{k} \text { for } n, k \geq 0 .
$$

It is clear that $F(n, k)=F_{n+k+2}$.

Definition 2.1. Let $G$ be a 2-generator group and let $(x, y)$ be a generating pair of $G$. The kth double Fibonacci orbit $F_{(x, y)}^{k}(G)=\left\{a_{n}^{k}\right\}$ of $(x, y)$ is defined by

$$
a_{n+2}^{k}=\left(a_{n+1}^{k}\right)^{F_{k}}\left(a_{n}^{k} a_{n+1}^{k}\right)^{F_{k+1}}, n \geq 1
$$

in which $a_{1}^{k}=x, a_{2}^{k}=y$ and $k \geq 1$.

Theorem 2.1. A double Fibonacci orbit of a finite 2-generator group is periodic.

Proof. Let $\alpha$ be order of the group G; then it is clear that there are $\alpha^{2}$ distinct 2-tuples of elements of G. Thus it is verified that at least one of the 2-tuples appears twice in a double Fibonacci orbit. Because of the repeating, the double Fibonacci orbit is periodic.

The period of the $k$ th double Fibonacci orbit $F_{(x, y)}^{k}(G)$ is denoted by $P F_{(x, y)}^{k}(G)$.

Definition 2.2. Let $k \geq 1$ be an integer and let $u$ be smallest positive integer such that $a_{u}^{k}=$ $a_{u+P F_{(x, y)}^{k}(G)}^{k}$ and $a_{u+1}^{k}=a_{u+P F_{(x, y)}^{k}(G)+1}^{k}$. For a generating pair $(x, y)$, the kth basic double Fibonacci orbit $\overline{F_{\left(b_{1}^{k}, b_{2}^{k}\right)}^{k}}(G)$ of the basic period $m$ is a sequence of group elements $b_{1}^{k}, b_{2}^{k}, \ldots, b_{n}^{k}, \ldots$ for which, given the initial (seed) set $b_{1}^{k}=a_{u}^{k}, b_{2}^{k}=a_{u+1}^{k}$, each element is defined by

$$
b_{n+2}^{k}=\left(b_{n+1}^{k}\right)^{F_{k}}\left(b_{n}^{k} b_{n+1}^{k}\right)^{F_{k+1}}, n \geq 1
$$

where $m \geq 2$ is the smallest integer with $b_{1}^{k}=b_{m+1}^{k} \theta$ and $b_{2}^{k}=b_{m+2}^{k} \theta$ for some $\theta \in$ Aut $G$ (where Aut $G$ means that the authomorphism group of $G$ ).

We denote the basic period of the $k$-th basic double Fibonacci orbit $\overline{F_{\left(b_{1}^{k}, b_{2}^{k}\right)}^{k}}(G)$ by

$$
\overline{\operatorname{BPF}_{\left(b_{1}^{k}, b_{2}^{k}\right)}^{k}}(G) \text {. }
$$

Theorem 2.2. Let $G$ be a finite 2-generator group and let $(x, y)$ be a generating pair of $G$. If $P F_{(x, y)}^{k}(G)=\lambda$ and $\overline{B P F_{\left(a_{u}^{k}, a_{u+1}^{k}\right)}^{k}}(G)=m$, then $m$ divides $\lambda$.

Proof. Let $u$ be the smallest positive integer such that $a_{u}^{k}=a_{u+P F_{(x, y)}^{k}(G)}^{k}$ and $a_{u+1}^{k}=a_{u+P F_{(x, y)}^{k}(G)+1}^{k}$. Then we have $\lambda=\eta \cdot m$ where $\eta$ is order of automorphism $\theta \in A$ At $G$ since

$$
F_{\left(a_{u}^{k}, a_{u+1}^{k}\right)}^{k}(G)=\overline{F_{\left(a_{u}^{k}, a_{u+1}^{k}\right)}^{k}}(G) \cup \overline{F_{\left(a_{u}^{k} \theta, a_{u+1}^{k} \theta\right)}^{k}}(G) \cup \overline{F_{\left(a_{u}^{k} \theta^{2}, a_{u+1}^{k} \theta^{2}\right)}^{k}}(G) \cup \cdots
$$

and

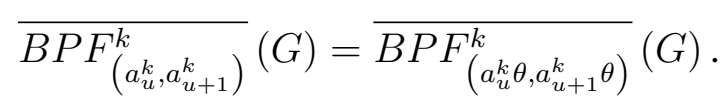


Now we give the periods of the $k$ th double Fibonacci orbits and the $k$ th basic double Fibonacci orbits of the dihedral group $D_{2 m}$ for $k \geq 1$ and $m \geq 3$ by the following Theorem.

Theorem 2.3. For $k \geq 1, m \geq 3$ and generating pair $(a, b)$, the periods of the $k$ th double Fibonacci orbits and the kth basic double Fibonacci orbits of the dihedral group $D_{2 m}$ are as follows:

(i). If $F_{k}$ and $F_{k+1}$ is odd, then $\overline{B P F_{(a, b)}^{k}}\left(D_{2 m}\right)=2$ and $P F_{(a, b)}^{k}\left(D_{2 m}\right)=4$.

(ii). If $F_{k}$ is even and $F_{k+1}$ is odd, then there are two subcases:

Case 1. If $F_{k+1} \mid m$, then $P F_{(a, b)}^{k}\left(D_{2 m}\right)=3$.

Case 2. If $F_{k+1} \nmid m$, then $\overline{B P F_{(a, b)}^{k}}\left(D_{2 m}\right)=3$ and $P F_{(a, b)}^{k}\left(D_{2 m}\right)=3 i$ where $i$ is the smallest positive integer such that:

$$
(-1)^{i}\left(F_{k+1}\right)^{i}=m \cdot r_{1}+1 \text { and }\left(-F_{k+1} F_{k}+F_{k+1}+1\right) \sum_{u=1}^{i}(-1)^{i-u}\left(F_{k+1}\right)^{i-u}=m \cdot r_{2}
$$

for $r_{1}, r_{2} \in \mathbb{Z}$.

(iii). If $F_{k}$ is odd and $F_{k+1}$ is even, then $P F_{(a, b)}^{k}\left(D_{2 m}\right)=2$.

Proof. (i). If $F_{k}$ and $F_{k+1}$ is odd, then the orbit $F_{(a, b)}^{k}\left(D_{2 m}\right)$ is

$$
a_{1}^{k}=a, a_{2}^{k}=b, a_{3}^{k}=a^{-1}, a_{4}^{k}=a^{-F_{k+1}+1} b, a_{5}^{k}=a, a_{6}^{k}=b, \ldots
$$

So, we get $\overline{B P F_{(a, b)}^{k}}\left(D_{2 m}\right)=2$ and $P F_{(a, b)}^{k}\left(D_{2 m}\right)=4$ since $a \theta=a^{-1}$ and $b \theta=a^{-F_{k+1}+1} b$ where $\theta$ is an automorphism of order 2 .

(ii). If $F_{k}$ is even and $F_{k+1}$ is odd, then we have the sequence

$$
\begin{aligned}
& a_{1}^{k}=a, a_{2}^{k}=b, a_{3}^{k}=a b, \\
& a_{4}^{k}=a^{-F_{k+1}}, a_{5}^{k}=a^{-F_{k+1} F_{k}+F_{k+1}+1} b, \ldots
\end{aligned}
$$

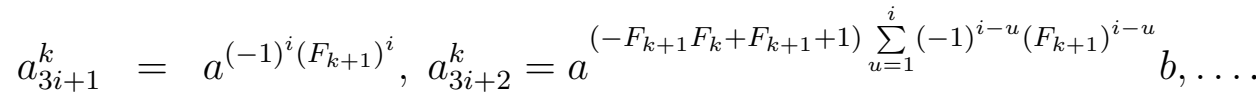

Thus, two cases occur:

Case 1. If $F_{k+1} \mid m$, then it is clear that $a_{3 i+1}^{k}=a_{3(i-1)+1}^{k}$ and $a_{3 i+1}^{k}=a_{3(i-1)+2}^{k}$. Thus, we obtain $P F_{(a, b)}^{k}\left(D_{2 m}\right)=3$.

Case 2. Let $F_{k+1} \nmid m$. If we choose $i$ as the smallest positive integer such that $(-1)^{i}\left(F_{k+1}\right)^{i}=$ $m \cdot r_{1}+1$ and $\left(-F_{k+1} F_{k}+F_{k+1}+1\right) \sum_{u=1}^{i}(-1)^{i-u}\left(F_{k+1}\right)^{i-u}=m \cdot r_{2}$ for $r_{1}, r_{2} \in \mathbb{Z}$, then we get $P F_{(a, b)}^{k}\left(D_{2 m}\right)=3 i$. Also, we obtain $\overline{\operatorname{BPF}_{(a, b)}^{k}}\left(D_{2 m}\right)=3$ since $a \theta=a^{\frac{-1}{F_{k+1}}}$ and $b \theta=$ $a^{\frac{-F_{k+1} F_{k}+F_{k+1}+1}{F_{k+1}}} b$ where $\theta$ is a automorphism of order $i$.

(iii). If $F_{k}$ is odd and $F_{k+1}$ is even, then we have the sequence

$$
a_{1}^{k}=a, a_{2}^{k}=b, a_{3}^{k}=b, a_{4}^{k}=b, a_{5}^{k}=b, \ldots,
$$

which has period 2. 
Theorem 2.4. For $k \geq 1, m \geq 3$ and generating pair $(b, a)$, the periods of the $k$ th double Fibonacci orbits and the kth basic double Fibonacci orbits of the dihedral group $D_{2 m}$ are as follows:

(i). If $F_{k}$ and $F_{k+1}$ is odd, then $\overline{B P F_{(b, a)}^{k}}\left(D_{2 m}\right)=2$ and $P F_{(b, a)}^{k}\left(D_{2 m}\right)=4$.

(ii). If $F_{k}$ is even and $F_{k+1}$ is odd, then two subcases occur:

Case 1. If $F_{k+1} \mid m$, then $P F_{(b, a)}^{k}\left(D_{2 m}\right)=3$.

Case 2. If $F_{k+1} \nmid m$, then $\overline{B P F_{(b, a)}^{k}}\left(D_{2 m}\right)=3$ and $P F_{(b, a)}^{k}\left(D_{2 m}\right)=3 i$ where $i$ is the smallest positive integer such that $\left(F_{k}\right) \sum_{v=1}^{i}(-1)^{i-u}\left(F_{k+1}\right)^{i-u}=m \cdot t_{1}$ and $(-1)^{i}\left(F_{k+1}\right)^{i}=m \cdot t_{2}+1$ and for $t_{1}, t_{2} \in \mathbb{Z}$.

(iii). If $F_{k}$ is odd and $F_{k+1}$ is even, then two subcases occur:

Case $1^{\prime}$. If $\operatorname{gcd}\left(F_{k+1}, m\right)=1$ and $\alpha \nmid F_{k+1}$ with $F_{k} \equiv \alpha(\bmod m)$, then $P F_{(b, a)}^{k}\left(D_{2 m}\right)=$ $\left|\left\langle Q_{k}\right\rangle_{m}\right|$ where the matrix $Q_{k}$ is defined by

$$
Q_{k}=\left[\begin{array}{cc}
F_{k+2} & F_{k+1} \\
1 & 0
\end{array}\right]
$$

Case 2'. If $\operatorname{gcd}\left(F_{k+1}, m\right)=1$ and $\alpha \mid F_{k+1}$ with $F_{k} \equiv \alpha(\bmod m)$, then $P F_{(b, a)}^{k}\left(D_{2 m}\right)=\tau$ where $\tau$ is the smallest positive integer such that $\left(Q_{k}\right)^{\vartheta} \equiv\left(Q_{k}\right)^{\vartheta-\tau}(\bmod m)$ for $\vartheta \in \mathbb{N}$.

Proof. The proofs of (i) and (ii) are similar to the above and are omitted. We will now prove only the condition (iii). When $F_{k}$ is odd and $F_{k+1}$ is even, we obtain the orbit $F_{(a, b)}^{k}\left(D_{2 m}\right)$ as follows:

$$
a_{1}^{k}=b, a_{2}^{k}=a, a_{3}^{k}=a^{F_{k}}, \ldots
$$

Consider the sequence

$$
v_{1}=x, v_{2}=F_{k}, v_{n+2}=v_{n+1}+F_{k+1}\left(v_{n+1}+v_{n}\right), n \geq 1 \text {. }
$$

It is clear that $P F_{(b, a)}^{k}\left(D_{2 m}\right)$ is equal to the peroid of the sequence $\left\{v_{n}\right\}$ when read modulo $m$.

Let

$$
\left(Q_{k}\right)^{n}=\left[\begin{array}{ll}
q_{1,1}^{(k, n)} & q_{1,2}^{(k, n)} \\
q_{2,1}^{(k, n)} & q_{2,2}^{(k, n)}
\end{array}\right],
$$

then by induction on $n$, it is easy to see that $q_{1,1}^{(k, n)}+q_{1,2}^{(k, n)}=v_{n+2}$ and $q_{2,1}^{(k, n)}+q_{2,2}^{(k, n)}=v_{n+1}$. Thus, two cases occur:

Case $1^{\prime}$. If $\operatorname{gcd}\left(F_{k+1}, m\right)=1$ and $\alpha \nmid F_{k+1}$ with $F_{k} \equiv \alpha(\bmod m)$, then we easily see that the period of the sequence $\left\{v_{n}\right\}$ when read modulo $m$ is equal to the order of the cyclic group $\left\langle Q_{k}\right\rangle_{m}$.

Case $2^{\prime}$. If $\operatorname{gcd}\left(F_{k+1}, m\right)=1$ and $\alpha \mid F_{k+1}$ with $F_{k} \equiv \alpha(\bmod m)$, then the peroid of the sequence $\left\{v_{n}\right\}$ when read modulo $m$ is the smallest positive integer $\tau$ such that $\left(Q_{k}\right)^{\vartheta} \equiv$ $\left(Q_{k}\right)^{\vartheta-\tau}(\bmod m)$ for $\vartheta \in \mathbb{N}$.

So, the proof is complete. 
Definition 2.3. Let $R$ be a 2-generator ring and let $(x, y)$ be a generating pair of $R$. The double Fibonacci orbit of the ring $R$ with respect to the generating pair $(x, y)$, written $F_{(x, y)}(R)$, is the sequence

$$
x_{1}=x, x_{2}=y, x_{n+2}=(x+y) x_{n}+y x_{n+1}, n \geq 1 .
$$

Theorem 2.5. If $R$ is a finite 2-generator ring and $(x, y)$ is a generating pair of $R$, then the sequence $F_{(x, y)}(R)$ is periodic.

Proof. The proof is similar the proof of Theorem 2.1 and is omitted.

The period of the sequence $F_{(x, y)}(R)$ is denoted by $P F_{(x, y)}(R)$.

Definition 2.4. Let $u$ be smallest positive integer such that $x_{u}=x_{u+P F_{(x, y)}(R)}$ and $x_{u+1}=$ $x_{u+P F_{(x, y)}(R)+1}$. For a generating pair $(x, y)$, the basic double Fibonacci orbit $\overline{F_{\left(c_{1}, c_{2}\right)}}(R)$ of the basic period $m$ is a sequence of ring elements $c_{1}, c_{2}, \ldots, c_{n}, \ldots$ for which, given the initial (seed) set $_{1}=x_{u}, c_{2}=x_{u+1}$, each element is defined by

$$
c_{n+2}=(x+y) c_{n}+y c_{n+1}, n \geq 1
$$

where $m \geq 2$ is the smallest integer with $c_{1}=c_{m+1} \theta$ and $c_{2}=c_{m+2} \theta$ for some $\theta \in$ Aut $R$ (where Aut $R$ means that the set of all authomorphisms of the ring $R$ ).

We denote the period of the sequence $\overline{F_{\left(c_{1}, c_{2}\right)}}(R)$ by $\overline{B P F_{\left(c_{1}, c_{2}\right)}}(R)$. The period $\overline{B P F_{\left(c_{1}, c_{2}\right)}}(R)$ is called the basic double period of the ring $R$ with respect to the initial (seed) set $c_{1}, c_{2}$.

Theorem 2.6. Let $R$ be a finite 2-generator ring and let $(x, y)$ be a generating pair of $R$. If $P F_{(x, y)}(R)=\beta$ and $\overline{B P F_{\left(x_{u}, x_{u+1}\right)}}(R)=m$, then $m$ divides $\beta$.

Proof. The proof is similar the proof of Theorem 2.2 and is omitted.

In [6], Deveci and Karaduman denoted the peroid of the Pell sequence $\left\{P_{n}\right\}$ when read modulo $m$ by $h P_{2}(m)$. Gopalan and Geetha [8] derived a relation among the Pell sequence $\left\{P_{n}\right\}$ and the half-companion Pell sequence $\left\{H_{n}\right\}$ as follows:

$$
H_{n+1}=H_{n}+2 P_{n} .
$$

Thus, it is clear that $h P_{2}(m)$ is equal to the period of the sequence $\left\{H_{n}\right\}$ when read modulo $m$.

Theorem 2.7. (Deveci and Karaduman [6]). $h P_{2}(m)$ is an even number.

Now we give the periods of the double Fibonacci orbits and the basic double Fibonacci orbits of the ring $E$ by the aid of $h P_{2}(m)$.

Theorem 2.8. The lengths of the double Fibonacci periods and the basic double Fibonacci periods of the ring $E$ are as follows:

(i). For the generating pairs $(a, b)$ and $(b, a)$,

$$
P F_{(a, b)}(E)=P F_{(b, a)}(E)=h P_{2}(p) .
$$

(ii). For the initial (seed) sets $a+2 b, 3 a+4 b$ and $2 a+b, 4 a+3 b$,

$$
\overline{B F_{(a+2 b, 3 a+4 b)}}(E)=\overline{B F_{(2 a+b, 4 a+3 b)}}(E)=2 .
$$


Proof. (i). It is important to note that $p a=p b=0$. First, let us consider the double Fibonacci orbit $F_{(a, b)}(E)$. Then we have the sequence

$$
x_{1}=a, x_{2}=b, x_{3}=2 b+a, x_{4}=4 b+3 a, \ldots
$$

Using the above we obtain the subsequence:

$$
y_{1}=2 b+a, y_{2}=4 b+3 a, \ldots, y_{i}=2 P_{i} \cdot b+H_{1} \cdot a, \ldots
$$

Then, it is readily seen that the period of the sequence $\left\{y_{n}\right\}$ is $h P_{2}(p)$. So, we get

$$
P F_{(a, b)}(E)=h P_{2}(p) \text {. }
$$

Now we consider the period $P F_{(b, a)}(E)$. The the double Fibonacci orbit $F_{(b, a)}(E)$ is in the following form:

$$
x_{1}=b, x_{2}=a, x_{3}=2 a+b, x_{4}=4 a+3 b, \ldots
$$

Then we have the subsequence

$$
z_{1}=2 a+b, z_{2}=4 a+3 b, \ldots, z_{i}=2 P_{i} \cdot a+H_{1} \cdot b, \ldots
$$

So, we get $P F_{(b, a)}(E)=h P_{2}(p)$.

(ii). From the sequence $\left\{y_{n}\right\}$, we obtain $\overline{B F_{(a+2 b, 3 a+4 b)}}(E)=2$ since $a \theta=3 a+(3 p-4) b$ and $b \theta=(p-2) a+3 b$ where $\theta$ is an automorphism of order $\frac{h P_{2}(p)}{2}$. Similarly, from the sequence $\left\{z_{n}\right\}$, we get $\overline{B F_{(2 a+b, 4 a+3 b)}}(E)=2$ since $a \theta=3 a+(3 p-2) b$ and $b \theta=(3 p-4) a+3 b$ where $\theta$ is an automorphism of order $\frac{h P_{2}(p)}{2}$.

\section{References}

[1] Aydın, H. \& Dikici, R. (1998) General Fibonacci Sequences in Finite Groups, Fibonacci Quart., 36, 216-221.

[2] Buschman, R. G. (1963) Fibonacci Numbers, Chebyshev Polynomials, Generalizations and Difference Equations, Fibonacci Quart., 1(4), 1-7.

[3] Campbell, C. M., Campbell, P. P., Doostie, H. \& Robertson, E. F. (2004) Fibonacci Lengths for Certain Metacyclic Groups, Algebra Colloq., 11, 215-222.

[4] Campbell, C. M., Doostie, H. \& Robertson, E. F. (1990) Fibonacci Length of Generating Pairs in Groups, Applications of Fibonacci Numbers, Springer Netherlands, 27-35.

[5] DeCarli, D. J. (1970) A Generalized Fibonacci Sequence Over An Arbitrary Ring, Fibonacci Quart., 8(2), 182-184.

[6] Deveci, O. \& Karaduman, E. (2015) The Pell Sequences in Finite Groups, Util. Math., 96, 263-276. 
[7] Fine, B. (1993) Classification of Finite Rings of Order $p^{2}$, Math. Mag., 66(4), 248-252.

[8] Gopalan, M. A. \& Geetha, V. (2014) Observations on Half-Companion Pell-Numbers, Int. J. Sci. Res. Publ., 4(2), 1-6.

[9] Horadam, A. F. (1961) A Generalized Fibonacci Sequence, Amer. Math. Monthly, 68(5), 445-459.

[10] Karaduman, E. \& Aydın, H. (2004) On The Relationship Between The Recurrences in Nilpotent Groups and The Binomial Formula, Indian J. Pure Appl. Math., 35, 1093-1103.

[11] Ozkan, E. (2014) Truncated Lucas Sequence and Its Period, Appl. Math. Comput., 232, 285-291.

[12] Tasyurdu, Y. \& Deveci, O. (2017) The Fibonacci Polynomials in Rings, Ars Combinatoria, $133,355-366$.

[13] Vorobyov, N. N. (1963) The Fibonacci Numbers, Translated from Russian by Norman D. Whaland, J r., and Olga A. Tittlebaum, D. C. Heath and Co., Boston. 